\title{
Changes in Hyolaryngeal Movement and Swallowing Function After Neuromuscular Electrical Stimulation in Patients With Dysphagia
}

\author{
Hoo Young Lee, $\mathrm{MD}^{1,2}$, Ji Seong Hong, $\mathrm{MD}^{3}$, Kil Chan Lee, $\mathrm{MD}^{4}$, \\ Yoon-Kyum Shin, $\mathrm{MS}^{5}$, Sung-Rae Cho, MD, $\mathrm{PhD}^{2,4,5,6}$
}

\begin{abstract}
${ }^{1}$ Department of Rehabilitation Medicine, Gangnam Severance Hospital, Seoul; ${ }^{2}$ Rehabilitation Institute of Neuromuscular Disease, Yonsei University College of Medicine, Seoul; ${ }^{3}$ Department of Rehabilitation Medicine, National Health Insurance Service Ilsan Hospital, Goyang; ${ }^{4}$ Department of Rehabilitation Medicine and Research Institute of Rehabilitation Medicine, ${ }^{5}$ Brain Korea 21 PLUS Project for Medical Science, and ${ }^{6}$ Avison Biomedical Research Center, Yonsei University College of Medicine, Seoul, Korea
\end{abstract}

Objective To investigate immediate changes in hyolaryngeal movement and swallowing function after a cycle of neuromuscular electrical stimulation (NMES) on both submental and throat regions and submental placement alone in patients with dysphagia.

Methods Fifteen patients with dysphagia were recruited. First, videofluoroscopic swallowing study (VFSS) was performed before NMES. All patients thereafter received a cycle of NMES by 2 methods of electrode placement: 1) both submental and throat regions and 2) submental placement alone concomitant with VFSS. The PenetrationAspiration Score (PAS) and the NIH-Swallowing Safety Scale (NIH-SSS) were measured for swallowing function.

Results During swallowing, hyolaryngeal descent significantly occurred by NMES on both submental and throat regions, and anterior displacement of hyolaryngeal complex was significant on submental placement alone. NMES on submental placement alone did not change the PAS and NIH-SSS. However, NMES on both submental and throat regions significantly reduced the NIH-SSS, although it did not change the PAS. Patients with no brainstem lesion and with dysphagia duration of $<3$ months showed significantly improved the NIH-SSS.

Conclusion Immediate hyolaryngeal movement was paradoxically depressed after NMES on both submental and throat regions with significant reductions in the NIH-SSS but not the PAS, suggesting improvement in pharyngeal peristalsis and cricopharyngeal functions at the esophageal entry rather than decreased aspiration and penetration. The results also suggested that patients with dysphagia should be carefully screened when determining motor-level NMES.

Keywords Deglutition, Electric stimulation, Hyoid bone, Larynx

Received July 3, 2014; Accepted September 26, 2014

Corresponding author: Sung-Rae Cho

Department of Rehabilitation Medicine and Research Institute of Rehabilitation Medicine, Yonsei University College of Medicine, 50 Yonsei-ro, Seodaemun-gu, Seoul 120-749, Korea

Tel: +82-2-2228-3715, Fax: +82-2-363-2795, E-mail: srcho918@yuhs.ac

(c) This is an open-access article distributed under the terms of the Creative Commons Attribution Non-Commercial License (http://creativecommons.org/ licenses/by-nc/3.0) which permits unrestricted noncommercial use, distribution, and reproduction in any medium, provided the original work is properly cited. Copyright $\odot 2015$ by Korean Academy of Rehabilitation Medicine 


\section{INTRODUCTION}

Swallowing is a complex function that requires elevation and anterior excursion of the hyolaryngeal complex in the pharyngeal phase, which aids laryngeal vestibule closure and serves to prevent aspiration into the respiratory tract [1]. In recent years, surface electrical stimulation has been gaining attention for its muscle strengthening effect by motor stimulation and facilitation of swallowing reflex by sensory stimulation [2]. The effects of neuromuscular electrical stimulation (NMES) on the physiology of swallowing have been well studied but remain unclear. The emerging hypothesis on the physiological effects of NMES on swallowing is that motor stimulation produces a resistance to hyolaryngeal elevation and induces effortful swallowing [3]. Shaw et al. [4] retrospectively analyzed patients who received NMES with electrodes placed in both submental and throat regions, and demonstrated an improved swallowing function in patients with mild to moderate dysphagia. A cohort study by Blumenfeld et al. [5] suggested that dysphagia therapy with NMES on throat regions with a motor-level stimulation is superior to traditional dysphagia therapy alone in individuals in the acute care facility. On the other hand, Humbert et al. [6] designed a study with electrodes placed in different ways including both submental and throat regions and submental placement alone. Their results showed a significant hyolaryngeal descent with stimulation at rest and significant reduction in hyolaryngeal peak elevation during swallowing when the electrodes were placed in both submental and laryngeal regions with intensity at the motor level. It was suggested that in those patients who had the ability to raise their hyolaryngeal complex, hyoid depression with stimulation might serve as resistance exercise during therapy. However, if patients were unable to produce hyolaryngeal elevation and were unable to resist the NMES-induced hyoid depression, the stimulation might increase the risk of aspiration, as the hyolaryngeal complex would remain held down during swallowing. Stimulated swallows were less safe than non-stimulated swallows according to the National Institutes of Health-Swallowing Safety Scale (NIH-SSS). Other studies showed that NMES had no additional improvement, as compared to traditional swallowing treatments $[7,8]$. Thus, the mechanism underlying NMES therapy for dysphagia is still unclear.
Of the electrode placement methods of NMES, two different methods such as both submental and throat regions and submental placement alone have been widely used to investigate the actual strengthening effect $[2,6]$. NMES on both submental and throat regions has been theoretically expected to strengthen thyrohyoid muscle that contributes to elevation of hyolarynx. NMES with submental placement alone reinforces muscle complexes such as anterior belly of the digastric and mylohyoid that are anatomically responsible for hyoid elevation. However, more superficially located sternohyoid and omohyoid muscles that depress hyoid are also affected by NMES on both submental and throat regions, and geniohyoid muscle that pulls the hyoid anteriorly rather than hyoid elevation might be simultaneously affected by NMES with submental placement alone, respectively $[2,6]$.

We comparatively investigated immediate changes in hyolaryngeal movement and swallowing function induced by a cycle of NMES on both submental and throat regions and submental placement alone in patients with dysphagia. Furthermore, we investigated the changes before and after NMES according to the presence of brainstem lesion and duration of dysphagia.

\section{MATERIALS AND METHODS}

\section{Participants}

Fifteen patients were recruited for evaluation and management of their dysphagia. Because NMES is reportedly beneficial for swallowing in heterogeneous patient etiologies such as stroke, cancer, head trauma, and respiratory failure $[9,10]$, the inclusion criteria were dysphagia patients of variable etiologies including medical deconditioning. Patients with severe cognitive dysfunction or apraxia that precluded one command obey, exhibition of nonstop verbalization, significant reflux from the use of a feeding tube, dysphagia due to drug toxicity, agitation, decreased level of consciousness or otherwise noncompliant, and pregnancy were excluded [7]. Patients with poor sitting balance, or unstable medical condition were also excluded.

General characteristics of subjects were described in Table 1 . There were 9 male and 6 female patients. The mean age was 58 years old. Causes of dysphagia were diverse, i.e., 4 brain tumor, 3 stroke, 2 cerebral palsy, 1 traumatic brain injury, 3 Parkinsonism, and 2 medical 


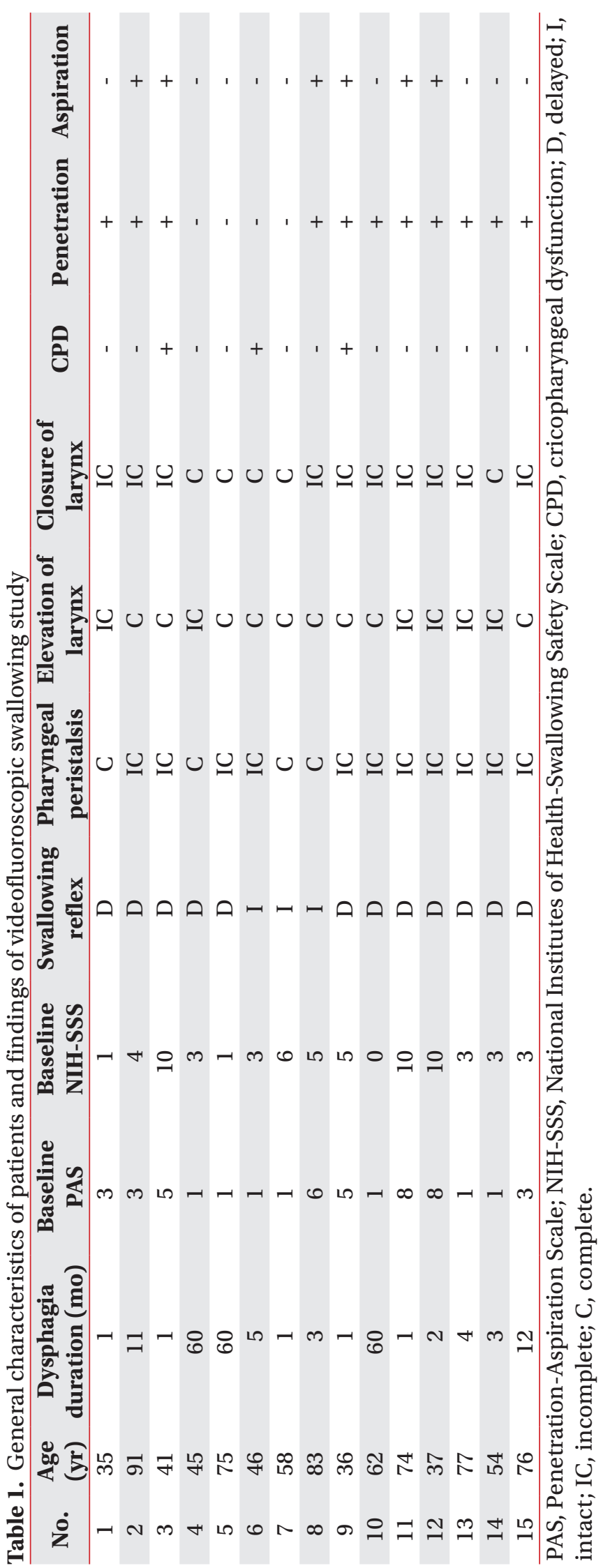

deconditioning without any brain lesion. Among the 13 participants who had a brain lesion, 4 patients had a brainstem lesion and 9 patients had no brainstem lesion. Six patients had dysphagia duration of $<3$ months and 9 patients had duration of $>3$ months. The mean duration of dysphagia was 3 months.

Since the aim of this study was to investigate the NMESinduced immediate changes of the hyolaryngeal movement, a subject who complained of dysphagia but did not show any abnormal findings in videofluorographic swallowing study (VFSS) before NMES and a subject who showed just delayed swallowing reflex and incomplete laryngeal elevation in VFSS were also included in the study.

\section{Procedure}

All VFSS procedures were conducted by 2 physiatrists and a radiologic technologist. First, VFSS was performed to measure the hyolaryngeal movement during nonstimulated swallow before NMES. The participants were seated upright laterally, and cough-induced head movements were stabilized with the examiner's hands. Distance from the X-ray tube to the laryngeal prominence was maintained at $0.5 \mathrm{~m}$. The videofluoroscopic image was recorded on a videocassette recorder at 10 frames per second. They were instructed to check movement of tongue and lip and elevation of velum, and to hold the $5 \mathrm{~mL}$ thin liquid of barium sulfate suspension in their mouth until told to swallow [11]. Subjects were then told to swallow without NMES. Particular attention was paid to the pharyngeal phase including presence of penetration and aspiration.

Each participant was subsequently familiarized with the sensations from the surface electrical stimulation unit (VitalStim; Chattanooga Group, Hixson, TN, USA). The electrical stimulation unit provided 2 channels of bipolar electrical stimulation at a fixed $80 \mathrm{~Hz}$ pulse rate and a fixed biphasic pulse duration of $700 \mu \mathrm{s}$. Each channel was independently adjustable between 0 and $25 \mu \mathrm{A}$ stimulation intensity. The skin in the submental and laryngeal regions was cleaned with alcohol and wiped to increase its adherence to the electrodes. Adult sized electrodes with a $2.1-\mathrm{cm}$ round active area were used.

Next, all patients received a cycle of repetitive NMES (10 times per 1 cycle) with two methods of electrode placement: 1) both submental and throat regions and 2) sub- 
mental placement alone [12]. Since the goal of this study was to investigate the immediate effect of NMES on the hyolaryngeal movement and swallowing function, only a cycle of NMES was provided in random order. VFSS was conducted while NMES was applied with the two methods.

Two pairs of electrodes were used for placement in both submental and throat regions, with the top pair placed horizontally in the submental region over the region of the mylohyoid muscle above the hyoid bone. The bottom pair was placed on the skin over the thyroid cartilage on either side of the midline over the region of the thyrohyoid muscle medial to the sternocleidomastoid muscle (Fig. 1A). For the submental placement alone method, both pairs of horizontally arranged electrode were placed in the skin overlying the submental region (Fig. 1B). The edge of the hyoid bone was detected by palpation. Prior to data recording, each electrode pair was placed on the skin and the stimulation intensity was gradually raised in a 1-mA step-wise fashion until the participant could first feel a tingling sensation. Then, the stimulation level was gradually increased to the maximum level that the participant could tolerate. The maximum tolerance levels, which were at least $10 \mathrm{~mA}$ in all participants, were determined and recorded for all electrode pairs in a placement simultaneously. The stimulator contained 2-sets of bipolar electrodes, and automatically cycled at on for 59 seconds and off for 1 second.

This procedure was repeated twice if aspiration was not observed. The consistency of hyolaryngeal movement and swallowing safety scores were confirmed in such subjects. The stimulation level was set at the maximum tolerance level, as instructed in the training manual for the use of electrical stimulation in the treatment of dysphagia [12].

\section{Data analysis}

The movement of the hyolaryngeal complex such as elevation, depression, or anterior displacement was measured in non-stimulated and stimulated trials. In brief, the anterior-superior margin of the hyoid bone, the anterior margin of the subglottic airway column that represents the larynx, and the anterior-inferior margin of the second and fourth cervical vertebral bodies were measured by the Image (Wayne Rasband, National Institutes of Health, Bethesda, MD, USA) application tool that utilizes various metric measurements. ImageJ is a public domain Java image processing and analysis program with widespread academic application [13]. We defined the zero point as the anterior-inferior margin of the fourth cervical vertebral body, and the y-axis as the straight line connecting the zero point and the anterior-inferior margin of the second cervical vertebral body. The $\mathrm{x}$-axis was drawn at a $90^{\circ}$ angle to the y-axis through the point on the fourth cervical vertebra. The maximal excursion point of the hyoid bone and the larynx during the swallowing reflex were analyzed in the $\mathrm{x}$ - and $\mathrm{y}$-axis by the ImageJ software.

We observed relative elevation or depression by subtracting the non-stimulated swallow peak shown as the absolute pixel number in $y$-axis from the stimulated swallow peak shown as another absolute pixel number in the same axis. Positive value meant relative elevation

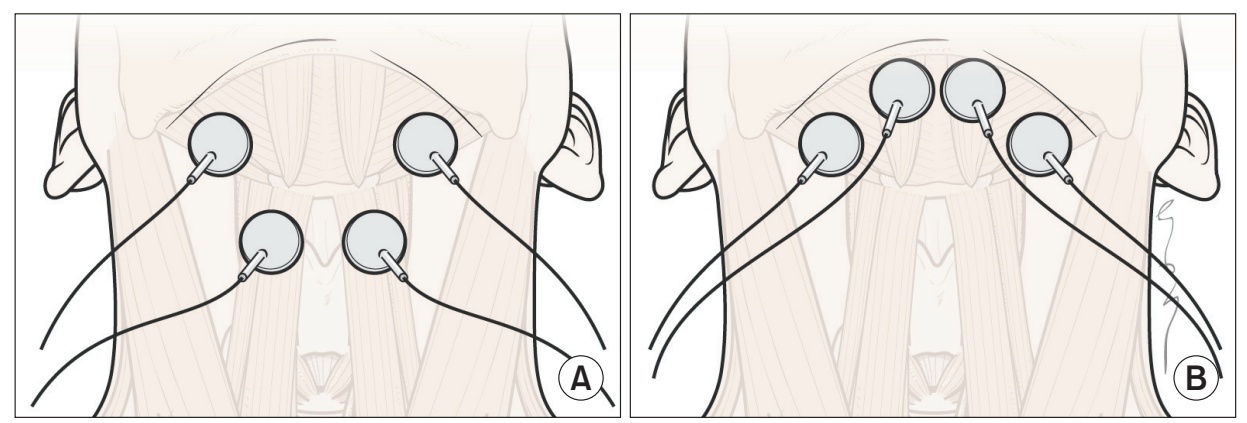

Fig. 1. The electrode positions relative to hyoid bone and thyroid cartilage. (A) Electrodes placement in both submental and throat regions. The top pair was placed horizontally in the submental region over the region of the mylohyoid muscle above the hyoid bone. The bottom pair was placed on the skin over the thyroid cartilage on either side of the midline over the region of the thyrohyoid muscle medial to the sternocleidomastoid muscle. (B) Electrodes in submental placement alone. Both pairs of horizontally arranged electrode were placed in the skin overlying the submental region. 
and negative value meant relative depression of the hyolaryngeal complex. The peak anterior displacement for the hyolaryngeal complex on the $\mathrm{x}$-axis was determined for non-stimulated and stimulated swallows for each subject. Relative anterior displacement of the hyolarynx was checked by subtracting the non-stimulated swallow peak shown as the absolute pixel number in $\mathrm{x}$-axis from the stimulated swallow peak shown as another absolute pixel number in the same axis. Negative value meant relative anterior movement of the hyolaryngeal complex. In this study, relative change in horizontal and vertical position of the hyolaryngeal complex during swallowing reflex was analyzed. Swallowing trials were also assessed for safety by two physiatrists using the Rosenbek Penetration-Aspiration Score (PAS) [14] and NIH-SSS (Table 2).

\section{Statistical analysis}

The primary goal of the study was to assess the immediate change in the hyolaryngeal movement and swallowing function in response to the NMES stimulation placements. McNemar test was used to compare changes between pre- and post-NMES on both submental and throat regions and submental placement alone, in order to test the hypothesis that surface electrical stimulation would cause descent or anterior displacement of hyolaryngeal complex. Wilcoxon signed-rank test for paired samples was also used to compare the PAS and NIH-SSS scores between non-stimulated and stimulated swallows. All statistical analyses were performed using the SPSS ver. 19.0 (IBM SPSS Inc., Armonk, NY, USA). A value of $\mathrm{p}<0.05$ was considered statistically significant.

\section{RESULTS}

\section{Findings of videofluoroscopic swallowing study}

Among 15 patients who complained of dysphagia, 12 patients (80\%) showed delayed swallowing reflex and 11 patients (73.3\%) showed incomplete pharyngeal peristalsis. Regarding the laryngeal movement, 6 patients $(40 \%)$ showed incomplete elevation of larynx and 10 patients (66.7\%) showed incomplete closure of larynx. In addition, 3 patients (20\%) showed cricopharyngeal dysfunction. Regarding the penetration and aspiration, 11 patients $(73.3 \%)$ showed penetration and 6 patients $(40 \%)$ showed aspiration (Table 1).

\section{Change in hyolaryngeal position after NMES}

Based on the previous findings that electrical stimulation applied to the anterior neck did not elevate hyolaryngeal complex but rather decreased hyolaryngeal excursion [2], we determined the comparative change in hyolaryngeal position during swallowing after NMES on both submental and throat regions versus submental placement alone.

When NMES was applied to both submental and throat regions $(n=15)$, a significant depression of the hyolaryngeal complex was observed during swallowing $(\mathrm{p}=0.016)$ (Table 3). However, there was no significant change in elevation or anterior excursion of the hyolaryngeal complex after NMES on both submental and throat regions. On the other hand, in the submental placement alone group $(n=15)$, there was a significant increase in the anterior excursion of hyolaryngeal complex $(\mathrm{p}=0.031)$ (Table

Table 2. Comparison of assessment items between PAS and NIH-SSS

\begin{tabular}{|c|c|c|}
\hline Assessment & PAS & NIH-SSS \\
\hline \multirow[t]{4}{*}{ Penetration } & Above the vocal folds, and ejected & From hypopharynx into the laryngeal vestibule \\
\hline & Above the vocal folds, and not ejected & From the pyriform into the laryngeal vestibule \\
\hline & Contact vocal folds, and ejected & - \\
\hline & Contact vocal folds, and not ejected & - \\
\hline \multirow[t]{3}{*}{ Aspiration } & Pass below vocal folds, ejected & Number of aspiration in each swallowing \\
\hline & $\begin{array}{l}\text { Pass below vocal folds, } \\
\text { not ejected despite effort }\end{array}$ & - \\
\hline & Pass below vocal folds, no effort to eject & - \\
\hline \multirow[t]{2}{*}{ Peristalsis } & - & Pooling in the vallecula \\
\hline & - & Pooling in the pyriform \\
\hline Cricopharyngeal function & - & Entering and clearing from the upper esophagus \\
\hline
\end{tabular}

PAS, Penetration-Aspiration Scale; NIH-SSS, National Institutes of Health-Swallowing Safety Scale. 


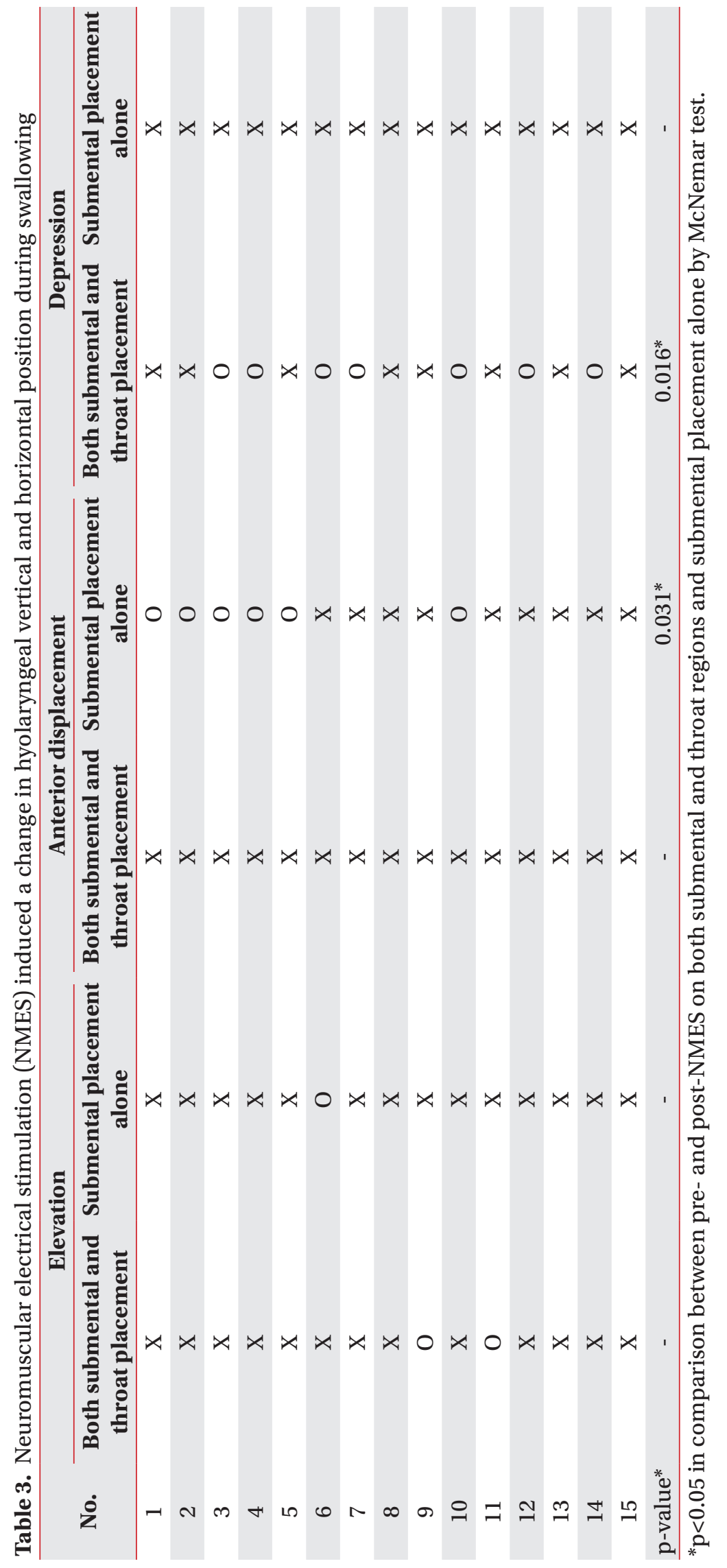


(A)

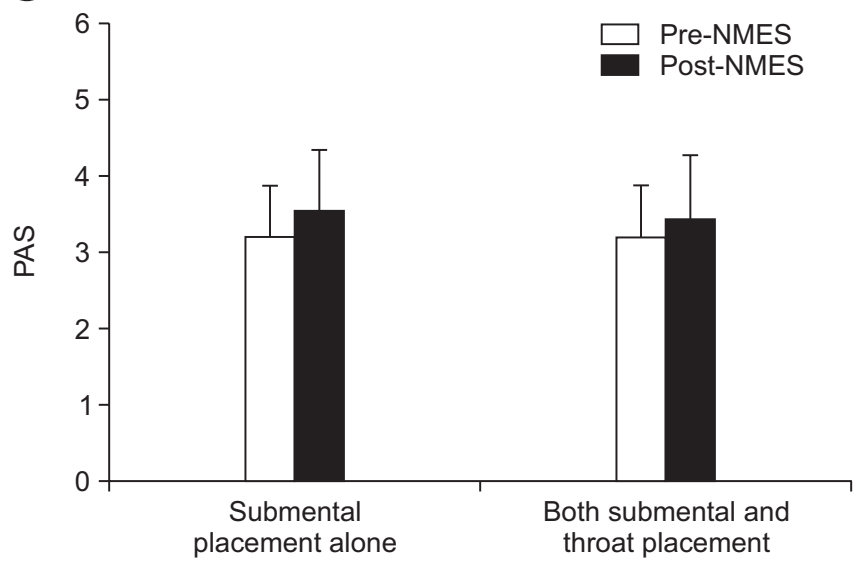

(B)

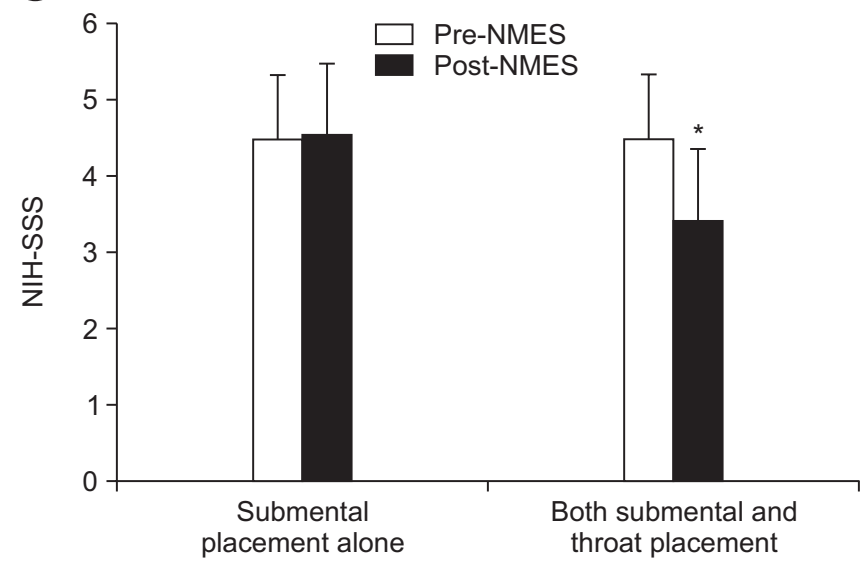

Fig. 2. Change in dysphagia rating scale after neuromuscular electrical stimulation. (A) There was no significant difference in the PAS after NMES on both submental and throat area and submental placement alone. (B) Application to both submental and throat regions significantly decreased the NIH-SSS $\left({ }^{*} \mathrm{p}<0.05\right)$, in contrast to the finding that submental placement alone did not change the dysphagia rating scores. PAS, Penetration-Aspiration Score; NIH-SSS, National Institutes of Health-Swallowing Safety Scale; NMES, neuromuscular electrical stimulation.

3). However, laryngeal elevation or depression was not significantly observed in the submental placement alone group. Taken together, neither NMES placements showed significant elevation of the hyolaryngeal complex.

\section{Change in dysphagia rating scale after NMES}

We compared the PAS and NIH-SSS after NMES on both submental and throat area stimulation versus submental placement alone to evaluate the risk for aspiration and swallowing safety scale in motor-level stimulation with two different placements.

The results indicated that there was no significant difference in the PAS after NMES on both submental and throat area stimulation and submental placement alone (Fig. 2A). However, application to both submental and throat regions significantly decreased the NIH-SSS from $4.47 \pm 0.84$ to $3.40 \pm 0.96$ ( $\mathrm{p}=0.027$ ), while submental placement alone did not change the dysphagia rating scores (Fig. 2B).

Change in dysphagia rating scale according to the presence of brainstem lesion

Swallowing reflex depends on swallowing centers in the brainstem, while initiation of swallowing is a voluntary action that involves the integrity of motor areas of the cerebral cortex $[15,16]$. Therefore, we compared the PAS and NIH-SSS on both submental and throat area stimulation and submental placement alone between patients with and without brainstem lesion.

There was no significant difference in the PAS after NMES on both submental and throat area stimulation and submental placement alone regardless of the brainstem lesion (Fig. 3A, B). In addition, patients with brainstem lesion did not show a significant change in the NIHSSS after NMES with both placement methods (Fig. 3D). However, patients with no brainstem lesion in whom NMES was applied to both submental and throat regions showed a significant decrease in the NIH-SSS from $4.36 \pm 0.98$ to $2.82 \pm 1.10$ ( $\mathrm{p}=0.026)$, whereas submental placement alone did not change the scores in the same patients (Fig. 3C).

Change in dysphagia rating scale according to the duration of dysphagia

We then determined whether the duration of dysphagia would affect the change in the dysphagia rating scale after NMES on both submental and throat area and submental placement alone. Given that chronic phase was defined as onset duration of $>3$ months [17], PAS and NIH-SSS were compared in patients with dysphagia duration that was $<3$ and $>3$ months.

As a result, there was no significant difference in the PAS after NMES on both submental and throat area and submental placement alone, irrespective of the duration of dysphagia (Fig. 4A, B). Additionally, subjects whose duration of dysphagia was $>3$ months did not show sig- 
(A)

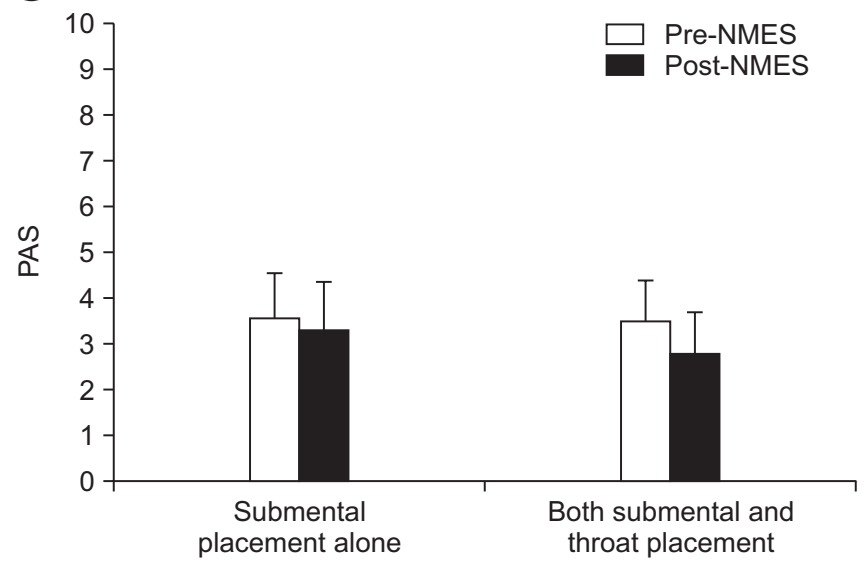

(C)

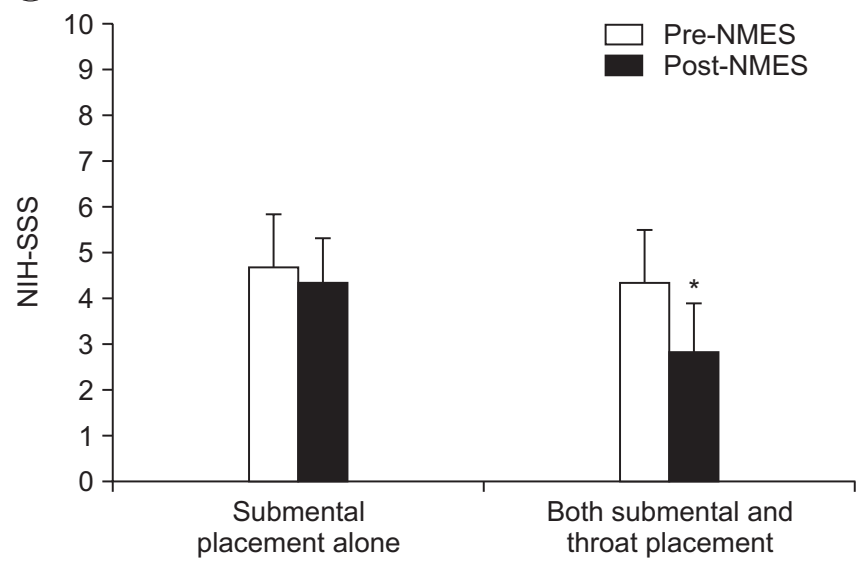

(B)

Brainstem lesion

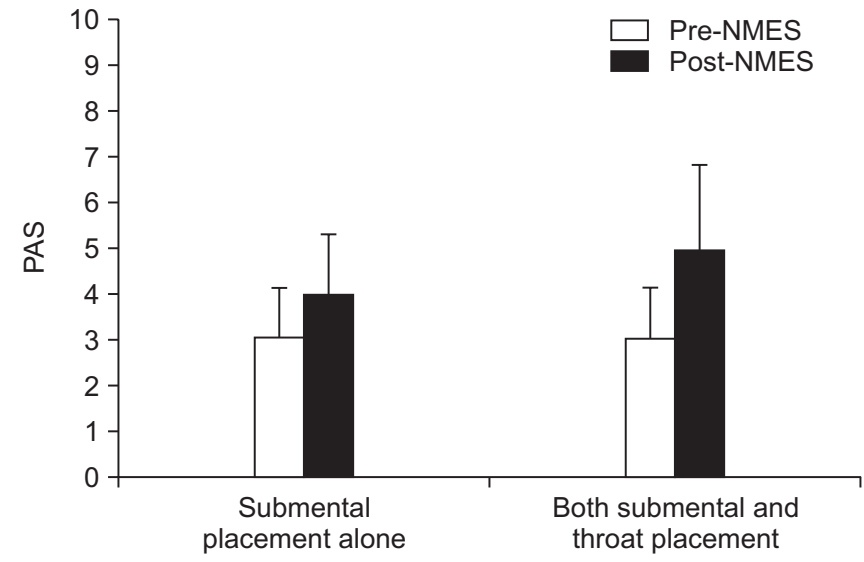

(D)

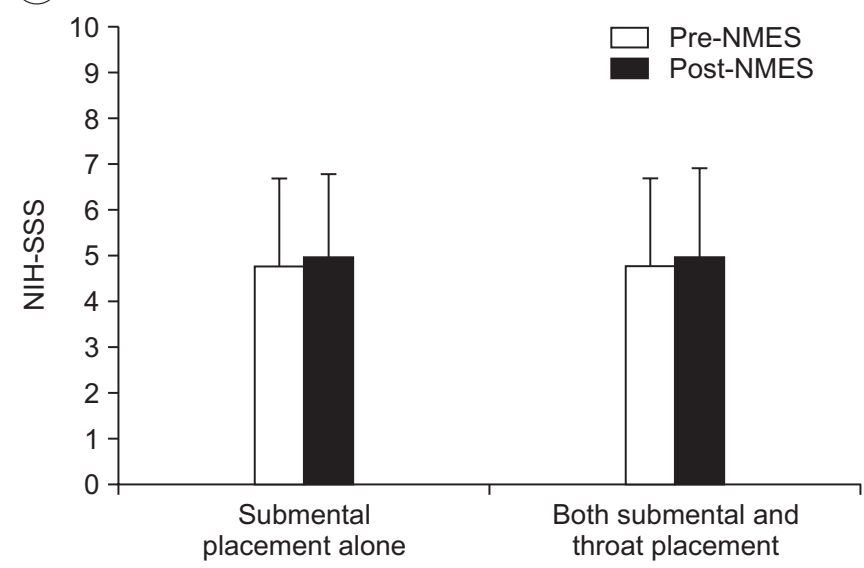

Fig. 3. Change in dysphagia rating scale according to the presence of brainstem lesion. (A, B) In the PAS, there was no significant difference after NMES on both submental and throat area and submental placement alone regardless of the brainstem lesion. (C) In the NIH-SSS, patients with no brainstem lesion in whom NMES was applied to both submental and throat regions showed significantly decreased scores $\left({ }^{*} \mathrm{p}<0.05\right)$, whereas submental placement alone did not change the scores in the same patients. (D) On the other hand, patients with brainstem lesion did not show significant change in the NIH-SSS after NMES in both placement methods. PAS, Penetration-Aspiration Score; NIH-SSS, National Institutes of Health-Swallowing Safety Scale; NMES, neuromuscular electrical stimulation.

nificant change in the NIH-SSS after NMES with both placement methods (Fig. 4D). On the other hand, patients whose dysphagia duration was $<3$ months showed a significant decrease in the NIH-SSS from $6.83 \pm 1.45$ to $2.83 \pm 1.52$ after NMES on both submental and throat area ( $\mathrm{p}=0.043)$, whereas submental placement alone did not change the scores in the same patients (Fig. 4C).

\section{DISCUSSION}

We investigated the immediate changes in hyolaryngeal movement and risk for aspiration and swallowing safety scale in response to NMES with placements on both submental and throat regions and the submental region alone in patients with dysphagia. In particular, this study showed that NMES on both submental and throat region significantly reduced the NIH-SSS, but not the PAS score, suggesting that pharyngeal peristalsis and cricopharyngeal functions at the esophageal entry might be improved rather than the decrease of aspiration and penetration.

During swallowing, the hyoid bone and larynx elevate by approximately $20 \mathrm{~mm}$ and the hyoid bone moves anteriorly by approximately $5 \mathrm{~mm}$ in healthy young males [18]. The suprahyoid muscles involved in hyolaryngeal elevation include the mylohyoid, geniohyoid, and anterior belly of the digastric muscles. The only muscle that 
(A)

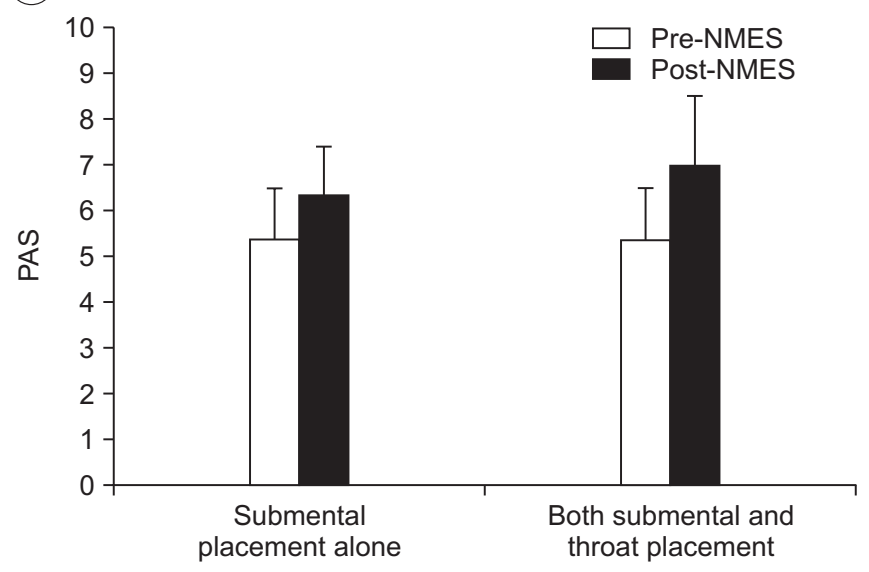

(C)

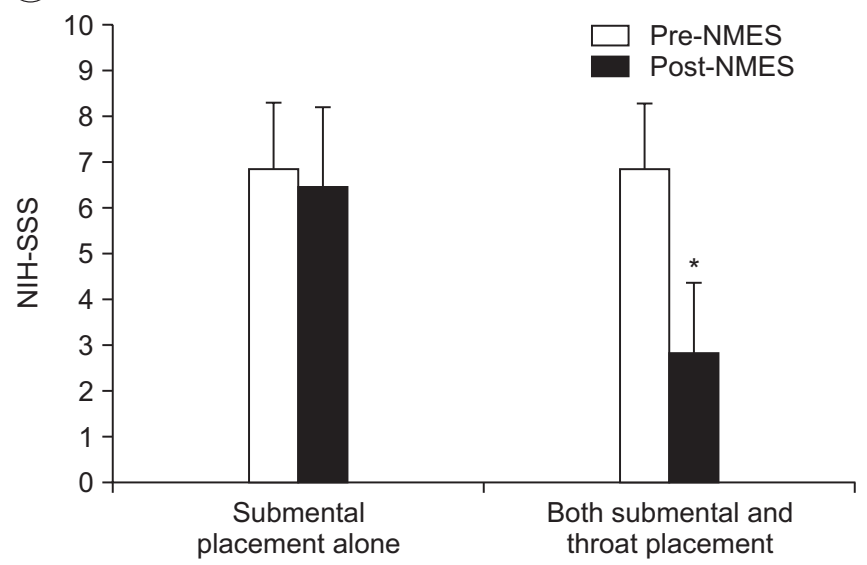

(B)

Dysphagia duration $\geq 3$ months

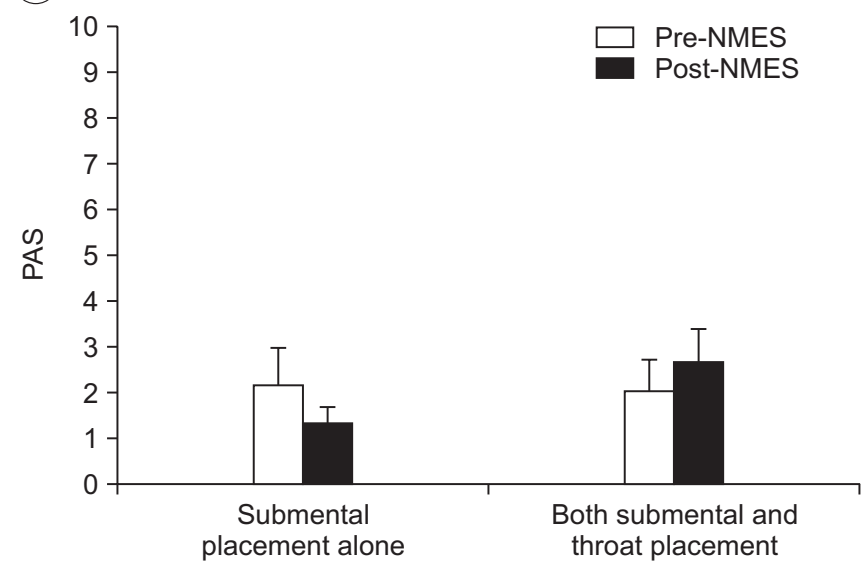

(D)

Dysphagia duration $\geq 3$ months

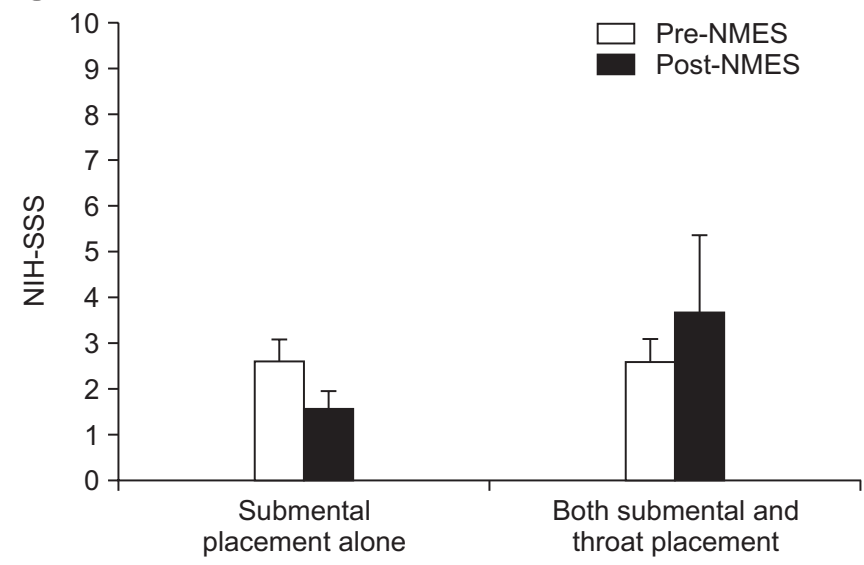

Fig. 4. Change in dysphagia rating scale according to the duration of dysphagia. (A, B) In the PAS, there was no significant difference after NMES on both submental and throat area and submental placement alone irrespective of the duration of dysphagia. (C) In the NIH-SSS, patients whose dysphagia duration was $<3$ months showed significant decrease in the NIH-SSS after NMES on both submental and throat area $\left({ }^{*} \mathrm{p}<0.05\right)$, whereas submental placement alone did not change the scores in the same patients. (D) On the other hand, subjects whose duration of dysphagia was $>3$ months did not show a significant change in the NIH-SSS after NMES in both placement methods. PAS, PenetrationAspiration Score; NIH-SSS, National Institutes of Health-Swallowing Safety Scale; NMES, neuromuscular electrical stimulation.

elevates the larynx to the hyoid is the thyrohyoid muscle, which lies beneath the strap muscles such as sternohyoid and omohyoid [19].

In previous studies by Ludlow et al. [2], hyoid depression was observed with motor-level stimulation on placement of surface electrodes over the anterior neck region. With respect to the relationship between the clinical safety rating scale and the depression of hyolaryngeal movement, no group change in aspiration scale was noted. Rather, levels of electrical stimulation just above the sensory threshold for detecting a tingling skin sensation, caused significant improvement during swallowing on the NIH-SSS.

Likewise, our results showed hyolaryngeal descent after motor-level stimulation on both submental and throat regions. This implies that sternohyoid and omohyoid stimulation exceeded the hyolaryngeal elevation effects. In other words, electrodes over the anterior neck might activate the sternohyoid and omohyoid rather than thyrohyoid that underlies the strap muscles and suprahyoid muscles such as geniohyoid and mylohyoid.

Submental stimulation alone on the surface of the skin produced no elevation of the hyolaryngeal complex but significant anterior movement. The anterior belly of the 
digastric muscle raises the hyoid if the jaw is clenched. Further below, the mylohyoid muscle moves the hyoid upwards to the mandible. Geniohyoid muscle lies deeper and pulls the hyoid bone forward towards the inside of the mandible [2]. If the currents run deep beneath the skin, NMES with submental placement alone may elevate the hyoid upwards in an anterior direction. However, the stimulation of the platysma without simultaneous stimulation of the thyrohyoid would leave the larynx in an anterior direction, possibly resulting in further opening of the vestibule and increased aspiration risk. In this study, the PAS and NIH-SSS results indicated no significant change during swallowing with motor-level NMES on submental placement alone.

None of the previous studies have compared immediate changes in swallowing function between both submental and throat stimulation and submental stimulation alone. Our results showed that motor-level stimulation on the anterior neck area would lower the hyolaryngeal complex. Nevertheless, NMES on both submental and throat regions improved the NIH-SSS, although the PAS was unchanged. Especially, patients with no brainstem lesion and with dysphagia duration of $<3$ months had significantly improved NIH-SSS. This result suggested that the improved pharyngeal peristalsis and cricopharyngeal function at the esophageal entry would have more beneficial effects from NMES, rather than the decreased penetration and aspiration risks caused by elevation of the hyolaryngeal complex (Table 3).

A limitation of this study was that it included a small number of subjects with dysphagia of heterogeneous etiologies. Recruitment of more subjects from a homogenous disease population is required to statistically compare immediate changes in hyolaryngeal movement by NMES with both submental and throat regions versus submental placement alone. We also need to investigate the effects of long-term NMES on the hyolaryngeal movement and swallowing function for the treatment of dysphagia. In addition, as only relative change of hyolaryngeal movement rather than absolute distance to the maximal excursion was measured, further study measuring absolute distance is required. Moreover, as a single food preparation was used rather than foods with diverse viscosities, further study with diverse food textures needs to be conducted.

In conclusion, the study showed that a cycle of motor- level NMES on the submental and throat regions paradoxically caused immediate hyolaryngeal descent; and submental stimulation alone produced significant anterior displacement of the hyolaryngeal complex. However, NMES on both submental and throat regions significantly reduced the NIH-SSS, but not the PAS, suggesting pharyngeal peristalsis and cricopharyngeal functions at the esophageal entry might be improved rather than the decrease of aspiration and penetration. The results also suggested that patients with dysphagia should be carefully screened before planning motor-level NMES for dysphagia treatment.

\section{CONFLICT OF INTEREST}

No potential conflict of interest relevant to this article was reported.

\section{ACKNOWLEDGMENTS}

The work was supported by the National Research Foundation (NRF-2010-0020408, 2014R1A2A1A11052042) funded by the Ministry of Education, Science and Technology, Republic of Korea.

\section{REFERENCES}

1. Ertekin C, Aydogdu I. Neurophysiology of swallowing. Clin Neurophysiol 2003;114:2226-44.

2. Ludlow CL, Humbert I, Saxon K, Poletto C, Sonies B, Crujido L. Effects of surface electrical stimulation both at rest and during swallowing in chronic pharyngeal dysphagia. Dysphagia 2007;22:1-10.

3. Park JW, Oh JC, Lee HJ, Park SJ, Yoon TS, Kwon BS. Effortful swallowing training coupled with electrical stimulation leads to an increase in hyoid elevation during swallowing. Dysphagia 2009;24:296-301.

4. Shaw GY, Sechtem PR, Searl J, Keller K, Rawi TA, Dowdy E. Transcutaneous neuromuscular electrical stimulation (VitalStim) curative therapy for severe dysphagia: myth or reality? Ann Otol Rhinol Laryngol 2007;116:36-44.

5. Blumenfeld L, Hahn Y, Lepage A, Leonard R, Belafsky PC. Transcutaneous electrical stimulation versus traditional dysphagia therapy: a nonconcurrent cohort study. Otolaryngol Head Neck Surg 2006;135:754-7. 
6. Humbert IA, Poletto CJ, Saxon KG, Kearney PR, Crujido L, Wright-Harp W, et al. The effect of surface electrical stimulation on hyolaryngeal movement in normal individuals at rest and during swallowing. J Appl Physiol (1985) 2006;101:1657-63.

7. Kiger M, Brown CS, Watkins L. Dysphagia management: an analysis of patient outcomes using VitalStim therapy compared to traditional swallow therapy. Dysphagia 2006;21:243-53.

8. Bulow M, Speyer R, Baijens L, Woisard V, Ekberg O. Neuromuscular electrical stimulation (NMES) in stroke patients with oral and pharyngeal dysfunction. Dysphagia 2008;23:302-9.

9. Tan C, Liu Y, Li W, Liu J, Chen L. Transcutaneous neuromuscular electrical stimulation can improve swallowing function in patients with dysphagia caused by non-stroke diseases: a meta-analysis. J Oral Rehabil 2013;40:472-80.

10. Carnaby-Mann GD, Crary MA. Examining the evidence on neuromuscular electrical stimulation for swallowing: a meta-analysis. Arch Otolaryngol Head Neck Surg 2007;133:564-71.

11. Palmer JB, Kuhlemeier KV, Tippett DC, Lynch C. A protocol for the videofluorographic swallowing study. Dysphagia 1993;8:209-14.
12. Wijting Y, Freed ML. VitalStim therapy training manual. Hixson, TN: Chattanooga Group; 2003. p. 103

13. Abramoff MD, Magalhaes PJ, Ram SJ. Image processing with ImageJ. Biophotonics Int 2004;11:36-43.

14. Rosenbek JC, Robbins JA, Roecker EB, Coyle JL, Wood JL. A penetration-aspiration scale. Dysphagia 1996;11:93-8.

15. Hamdy S, Rothwell JC, Aziz Q, Thompson DG. Organization and reorganization of human swallowing motor cortex: implications for recovery after stroke. Clin Sci (Lond) 2000;99:151-7.

16. Hamdy S, Rothwell JC. Gut feelings about recovery after stroke: the organization and reorganization of human swallowing motor cortex. Trends Neurosci 1998;21:278-82.

17. Bath PM, Bath FJ, Smithard DG. Interventions for dysphagia in acute stroke. Cochrane Database Syst Rev 1999;4.

18. Jacob P, Kahrilas PJ, Logemann JA, Shah V, Ha T. Upper esophageal sphincter opening and modulation during swallowing. Gastroenterology 1989;97:1469-78.

19. Ludlow CL. Electrical neuromuscular stimulation in dysphagia: current status. Curr Opin Otolaryngol Head Neck Surg 2010;18:159-64. 